\title{
Thermal conductivity of aerated concrete (AC) composites containing micro-encapsulated phase change materials
}

\author{
Shuai-Qi Tian ${ }^{1}$, Ming-Liang $\mathrm{Qu}^{1}$, Li-Wu Fan ${ }^{1}, Z i-$ Tao $\mathrm{Yu}^{1,2, *^{*}}$ and Jian $\mathrm{Ge}^{3}$ \\ ${ }^{1}$ Institute of Thermal Science and Power Systems, School of Energy Engineering, Zhejiang \\ University, Hangzhou, Zhejiang 310027, People's Republic of China \\ ${ }^{2}$ State Key Laboratory of Clean Energy Utilization, Zhejiang University, Hangzhou, Zhejiang \\ 310027, People's Republic of China \\ ${ }^{3}$ Institute of Building Technology, School of Civil Engineering and Architecture, Zhejiang University, \\ Hangzhou 310058, People's Republic of China
}

\begin{abstract}
Aerated concrete (AC) was incorporated with microencapsulated phase change materials (PCMs) to form a novel PCMcomposite AC with improved thermal storage capacity. RT25 paraffin was selected as the PCM and the composite materials were prepared by adding micro-encapsulated paraffin as an ingredient at various loadings. The effective thermal conductivity of the composite samples was measured at both $17{ }^{\circ} \mathrm{C}$ and $35^{\circ} \mathrm{C}$, while the paraffin was in solid and liquid phases, respectively. The volumetric heat capacity of the composites were also measured. Results showed that both the thermal conductivity and volumetric heat capacity increase upon adding the micro-encapsulated paraffin. However, they were found to decrease when further increase the paraffin loading. The maximum thermal conductivity and volumetric heat capacity were enhanced by approximately $35 \%$ and $30 \%$ when the paraffin loadings were $1 \%$ wt. and $3 \%$ wt., respectively. Since the increase of thermal conductivity leads to the deterioration of the thermal insulation performance, the composite samples with 3\% wt. micro-encapsulated paraffin with lower thermal conductivity but the highest volumetric heat capacity was exhibited to be more appropriate.
\end{abstract}

\section{Introduction}

Embedding phase change materials (PCMs) in porous building materials has been proposed as a passive means to improve the thermal mass of building envelopes and reduce building energy consumption [1-3]. Generally, there are several typical ways to incorporate PCMs into porous building materials such as concretes. One is the direct immersion method by directly soaking liquid PCM into porous materials $[4,5]$. The second is impregnating PCM with some raw materials prior to the process of synthesis [6-8]. The third is incorporating micro-encapsulated PCM at the time of mixing [9]. Among all these methods, the microencapsulated PCM was utilized by incorporating the PCM into small polymeric capsules

*Corresponding author: yuzitao@zju.edu.cn 
[10]. It can prevent the leakage of PCM and the interactions with the building structure, and is therefore able to support PCM to be utilized as thermal storage materials integrated into building envelope materials [11]. As a lightweight porous building material, aerated concrete (AC) is widely utilized in building envelopes [12]. With high porosity and mechanical strength, AC is proved to be a potential candidate to integrate with $\mathrm{PCM}$, and the incorporation of PCM into AC can significantly improve the thermal energy storage capacity of the composite material [13]. However, in previous researches, there is a lack of investigation on AC samples integrated with PCM. Furthermore, their thermal conductivity will likely increase because the thermal conductivity of paraffin is higher than that of air, leading to deterioration of the thermal insulation performance. Therefore, the knowledge on the thermal conductivity and heat storage capacity of composite samples is of great significance $[14,15]$.

In this work, the integration of micro-encapsulated PCM into AC was investigated. The AC samples with high porosity was synthesized by adding aluminium powder of certain content into cement mortar composites as foaming agent. A new type of PCM-composite AC was synthesized by adding microencapsulated paraffin into AC. RT25 paraffin with an appropriate melting temperature of $25 \pm 1{ }^{\circ} \mathrm{C}$ was selected as the original PCM. The microencapsulated PCM was added with the mass fraction varies from 0 to $5 \mathrm{wt} . \%$. The effective thermal conductivity and volumetric heat capacity of composite AC with various fractions of microcapsules was measured through transient plane source (TPS) method. The effects of embedding microencapsulated paraffin inclusions on the thermal conductivity and heat capacity of PCM-composites were quantified. The results in this work can be used to guide the design of PCM-composite AC for energy efficient buildings.

\section{Sample preparation and thermal conductivity measurement}

\subsection{Preparation of paraffin/AC composite materials}

The AC samples with and without incorporating micro-encapsulated paraffin were investigated in this study. The micro-encapsulated paraffin/AC samples were prepared using a non-autoclave approach, which means the samples were cured in a climate chamber under an atmosphere pressure rather than an autoclave. The raw materials for fabricating a concrete sample, including cement, lime, kaolin, gypsum and coal ash were prepared. The proportions of each component are shown in Table 1. The mass of these components is defined as the matrix mass $m_{\mathrm{m}}$. Moreover, some other supporting materials were also needed, such as the aluminium powder as a foaming agency, sodium sulfate as booster and triethanolamine as foam stabilizer, and they are expected to be added with a certain mass fraction based on the matrix mass. In this work, the mass fractions of aluminium powder, sodium and triethanolamine were $0.5 \%$ wt., $0.48 \%$ wt. and $0.24 \%$ wt., respectively. Moreover, the mass fraction of micro-encapsulated paraffin was varied from 0 to $5 \%$, which is the main variable to be focused. RT25 paraffin with a melting temperature of $25 \pm$ $1{ }^{\circ} \mathrm{C}$ were selected.

Table 1. Mass fractions of each basic components for fabricating concrete samples.

\begin{tabular}{|c|c|c|c|c|c|}
\hline Element & Cement & Lime & Kaolin & Gypsum & Coal ash \\
\hline Proportion (\%) & 30 & 8 & 10 & 2 & 50 \\
\hline
\end{tabular}

The composite samples were cast into a square mould with a side length of $50 \mathrm{~mm}$. Then they were cured in sealed humid conditions with temperature and relative humidity being 
$85 \pm 0.5^{\circ} \mathrm{C}$ and $85 \pm 1.2 \% \mathrm{RH}$ for approximately one week [16]. For each microencapsulated paraffin content, three pairs of specimens were fabricated with the same procedure, which is required in the thermal conductivity measurement. In addition, prior to the measurement of thermal conductivity, some basic properties of composite samples, such as density, porosity and compressive strength were also measured.

\subsection{Measurement of thermal conductivity of composite materials}

Having fabricated the micro-encapsulate paraffin/AC composite samples, the thermal conductivity of the composite materials is of great significance to be revealed. In this paper, a transient approach, the transient plane source (TPS) method was utilized to measure the thermal conductivity of samples both with and without containing micro-encapsulated paraffin. A Hot Disk TPS 3500 Thermal Constants Analyzer was utilized, which is capable of measuring thermal conductivity over the range of $0.001 \sim 500 \mathrm{~W} /(\mathrm{m} \cdot \mathrm{K})$ with an accuracy of $5 \%$. The thermal conductivities of samples with different content of micro-encapsulated paraffin were measured both in the ambient temperature conditioned at $17 \pm 0.1{ }^{\circ} \mathrm{C}$ and 35 $\pm 0.1{ }^{\circ} \mathrm{C}$ to ensure the paraffin is in solid and liquid phases, respectively. Three pairs of specimens were fabricated with the same procedure and were tested independently in the same condition, and the measurements were repeated at least 3 times for each test.

\section{Results and discussion}

\subsection{Basic properties of fabricated paraffin/AC composite materials}

The photographic and microscopic images one of the composite samples with $3 \% \mathrm{wt}$. mass fraction of micro-encapsulated paraffin are shown in Fig. 1, indicating that the micro paraffin capsules with various sizes, which are marked with red circles, are nearly distributed. Furthermore, with a magnification of 7000, we can observe small flakes of the micron size, which is the fundamental composite of aerated concrete pores [17].

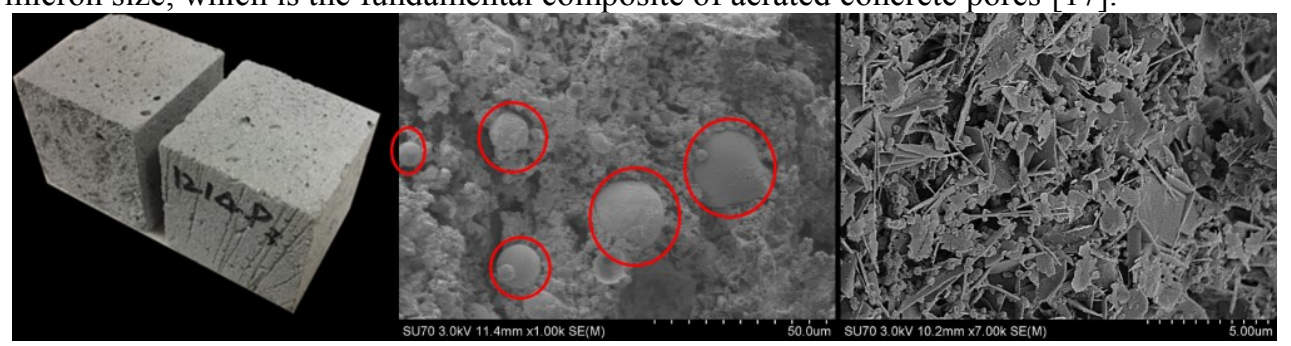

Fig. 1. Photographs and microscopic pore structures of paraffin/composite AC with different magnification.

The basic properties of composite materials were measured, which are listed in Table 2. We can see from Table 2 that for all types of composite materials, the porosity can be as high as approximately $60 \%$, which satisfies the standard of 'high porosity aerated concrete'. With the addition of the micro-encapsulated paraffin, the porosity decreases slightly while the bulk density increases. With increasing the content of the micro-encapsulated paraffin, the porosity increases and the bulk density decreases. We can also see from Fig. 2 showing the pore size distributions as determined by mercury intrusion porosimetry (MIP), there exists two dominant ranges of pore size. One is the mesoscale pores range from $5 \mathrm{~nm}$ to $50 \mathrm{~nm}$, another is the macroscale pores range from $0.05 \mathrm{~mm}$ to $0.5 \mathrm{~mm}$, which are the majority. The distributions of the mesoscale pores of various samples are nearly identical, whereas 
the macroscale pores varies with the content of micro-encapsulated paraffin, with the same trend as the porosity. This means the micro-encapsulated paraffin initially fills into the pores of the concrete, resulting in a decreasing porosity [18]. The further increase of the paraffin content will somehow cause a higher porosity, which also influences the compressive strength with the same trend as bulk density.

Table 2. The basic properties of concrete samples.

\begin{tabular}{|c|c|c|c|}
\hline $\begin{array}{c}\text { Mass fraction of micro- } \\
\text { encapsulated paraffin (\% wt.) }\end{array}$ & $\begin{array}{c}\text { Bulk density } \\
\left(\mathbf{k g} / \mathbf{m}^{\mathbf{3}}\right)\end{array}$ & Porosity (\%) & $\begin{array}{c}\text { compressive } \\
\text { strength (MPa) }\end{array}$ \\
\hline 0 & $494.4 \pm 13.1$ & $60.76 \pm 2.51$ & $4.10 \pm 0.47$ \\
\hline 1 & $597.6 \pm 14.3$ & $56.65 \pm 1.18$ & $9.60 \pm 0.10$ \\
\hline 3 & $546.4 \pm 19.5$ & $61.20 \pm 0.95$ & $6.35 \pm 1.00$ \\
\hline 5 & $476.0 \pm 21.3$ & $63.56 \pm 1.58$ & $5.48 \pm 0.20$ \\
\hline
\end{tabular}

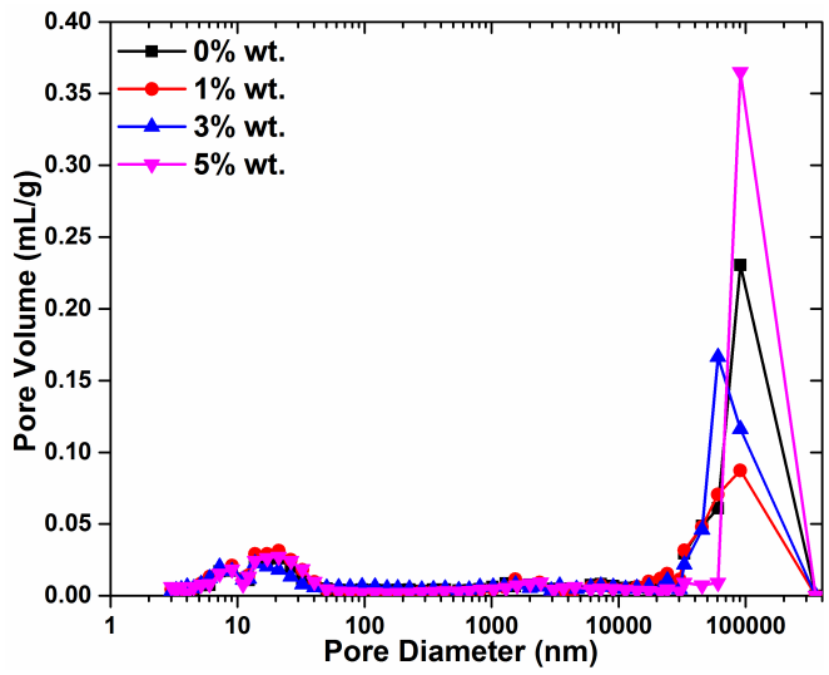

Fig. 2. Pore size distributions as determined by MIP.

\subsection{Measured paraffin content dependence on the thermal conductivity of composite samples}

The measured thermal conductivities of various composite materials were shown in Fig. 3 . The thermal conductivity of samples at $35^{\circ} \mathrm{C}$ was found to be higher than that at $17{ }^{\circ} \mathrm{C}$ as expected, this is due to the higher thermal conductivity of liquid paraffin. Moreover, as compared to samples without containing paraffin, the thermal conductivity was found to increase by approximately $35 \%$ when the content of micro-encapsulated paraffin is $1 \% \mathrm{wt}$.. When further increasing the paraffin content, the thermal conductivity becomes lower, when the paraffin content reaches $5 \%$ wt., only a $17 \%$ increase can be found as compared to the sample without paraffin. As introduced in section 3.1, we can know that the porosity for macroscale pores of various composite samples decreases when initially adding the micro-encapsulated paraffin, and the thermal conductivity of paraffin is about 12 times of dry air. Therefore, the thermal conductivity increases by a large scale. With further 
increasing the paraffin content, the porosity will be higher, which results in the decrease of the thermal conductivity.

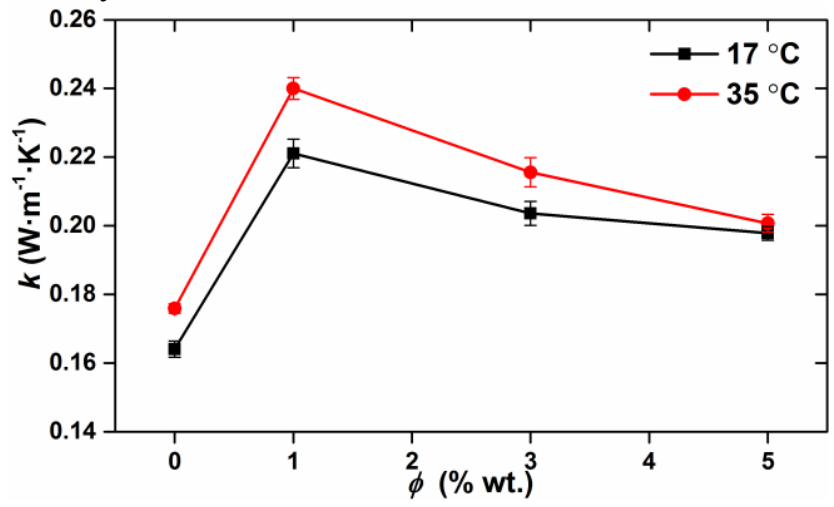

Fig. 3. Measured thermal conductivities of composite materials with various micro-encapsulated paraffin content.

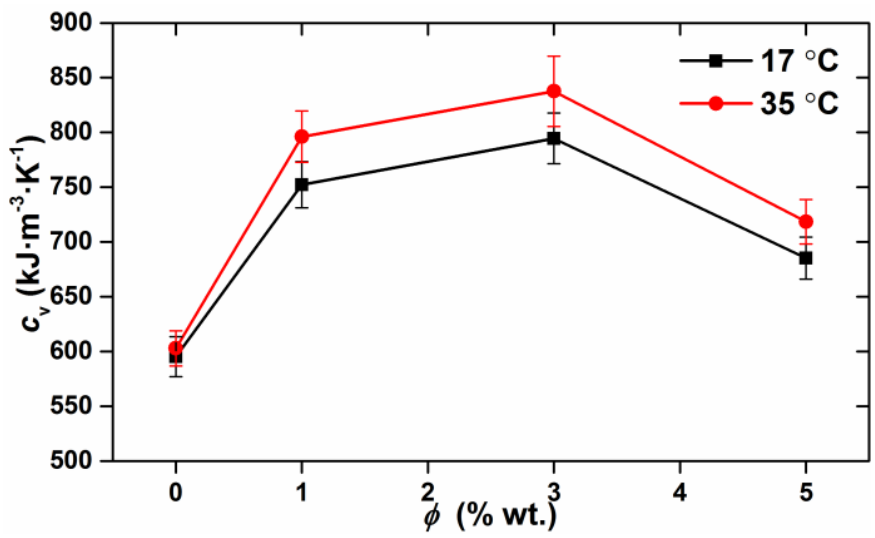

Fig. 4. Measured volume heat capacities of composite materials with various micro-encapsulated paraffin content.

The measured volumetric heat capacities of composite materials are shown in Fig. 4. Similar to the thermal conductivities, the volumetric heat capacity of composites with liquid paraffin capsules were found to be higher. The variation of heat capacity as increasing the content of micro-encapsulated paraffin was also similar to the thermal conductivity. However, the heat capacity was enhanced by approximately $30 \%$ with the content of micro-encapsulated paraffin being 3\% wt., which is the highest among all composite samples. In this point, the thermal storage capacity is the best with even better thermal insulation performance due to the relatively low thermal conductivity. As shown in Section 3.1, the bulk density, porosity and compressive strength of composites with a $3 \%$ wt. mass fraction of micro-encapsulated paraffin are also acceptable. Therefore, the composite samples with $3 \%$ wt. micro-encapsulated paraffin with lower thermal conductivity but the highest volumetric heat capacity was exhibited to be more appropriate to be utilized in building envelopes. 


\section{Conclusions}

In this paper, a new type of paraffin/AC composite samples was fabricated by containing micro-encapsulated paraffin with AC. The thermal conductivity as well as the volumetric heat capacity of composite materials with various contents of micro-encapsulated paraffin were investigated. When the paraffin turns to liquid phase, both the thermal conductivity and volumetric heat capacity were increased. Furthermore, they were found to increase when the micro-encapsulated paraffin was initially added but to decrease when further increase the content of paraffin capsule. When the contents of micro-encapsulated paraffin were $1 \%$ wt. and $3 \%$ wt. respectively, the thermal conductivity and volumetric heat capacity were found to be the highest. The composite samples with 3\% wt. microencapsulated paraffin with lower thermal conductivity but the highest volumetric heat capacity was proved to be the most appropriate to be utilized in building envelopes in this work. However, the conclusions only apply to the fabricated composite samples with a certain volume of aluminium powder, more measurements should be performed to determine more appropriate combinations of aluminium powder and micro-encapsulated paraffin.

This material is based upon work supported by the China National Key R\&D Program under Grant No. 2016YFC0700302.

\section{References}

1. S. Mirrahimi, M. F. Mohamed, L. C. Haw, N. L. N. Ibrahim, W. F. M. Yusoff, A. Aflaki, Renew. Sust. Energ. Rev. 53, 1508-1519 (2016)

2. N. P. Sharifi, A. A. N. Shaikh, A. R. Sakulich, Energ. Buildings 138, 455-467 (2017)

3. K. O. Lee, M. A. Medina, X. Sun, X. Jin, Sol. Energ. 163, 113-121 (2018)

4. D. W. Hawes, D. Feldman, A, Sol. Energ. Mat. Sol. C. 27, 91-101 (1992)

5. D. W. Hawes, D. Banu, D. Feldman, Sol. Energ. Mat. Sol. C 27, 103-118 (1992)

6. M. Hadjieva, R. Stoykov, T. Filipova, Renew. Energ. 19, 111-115 (2000).

7. A. Sari, A. Bicer, F. A. Al-Sulaiman, A. Karaipekli, V. V. Tyagi, Energ. Buildings 164, 166-175 (2018)

8. L. Jiang, Z. Liu, Y. Yuan, Y. Wang, J. Lei, C. Zhou, F, Energ. Buildings 171, 88-99 (2018)

9. V. V. Tyagi, A. K. Pandey, D. Buddhi, K. Richa, Energ. Buildings 117, 44-52 (2016).

10. M. Hunger, A.G. Entrop, I, Mandilaras, H.J.H Brouwers, M. Founti, Cement Concrete Comp. 31, 731-743 (2009)

11. A. Ricklefs, A.M. Thiele, G. Falzone, G. Sant, L. Pilon, Int. J. Heat Mass Tran. 104, 71-82 (2017)

12. N. Narayanan, K. Ramamurthy, Cement Concrete Comp. 22, 321-329 (2000)

13. D.W. Hawes, D. Feldman, D. Banu, Energ. Buildings 20, 77-86 (1993)

14. J. Shi, Z. Chen, S. Shao, J. Zheng, Appl. Therm. Eng. 66, 156-161 (2014)

15. Y. Wang, C. Ma, Y. Liu, D. Wang, J. Liu, Int. J. Heat Mass Tran. 125, 330-342 (2018)

16. C. Shi, D. Roy, Alkali-activated cements and concrete (CRC Press, Florida, 2006)

17. H.-Q. Jin, X.-L. Yao, L.-W. Fan, X. Xu, Z.-T. Yu, Int. J. Heat Mass Tran. 92, 589-602 (2016)

18. V. D. Cao, S. Pilehvar, C. Salas-Bringas, A. M. Szczotok, J. F. Rodriguez, M. Carmona, N. AlManasir, A.-L. Kjøniksen, Energ. Convers. Manage., 133, 56-66 (2017) 